\title{
Retrograde Abdominal Hysterectomy
}

\author{
Yuji Hiramatsu, MD, $\mathrm{PhD}^{1}$ \\ ${ }^{1}$ Okayama City General Medical Center, Okayama, Japan \\ Surg J 2019;5(suppl S1):S27-S32.
}

\begin{abstract}
Address for correspondence Yuji Hiramatsu, MD, PhD, Okayama City General Medical Center, 3-20-1 Kitanagase-Omotematchi, Kita-Ku, Okayama 700-8557, Japan (e-mail: kiki1063@cc.okayama-u.ac.jp).
\end{abstract}

\author{
Abstract \\ Keywords \\ - retrograde \\ hysterectomy \\ - adhesion \\ - large tumor mass in \\ the vagina \\ - uterine rupture
}

The basic procedure of abdominal hysterectomy is extrafascial hysterectomy, and intrafascial hysterectomy (Aldridge operation) and retrograde hysterectomy are performed as applied surgical procedures. The Aldridge operation and retrograde hysterectomy are performed when strong adhesion is present around the uterus. Retrograde hysterectomy is also useful when the cervicovaginal junction is not clearly felt by palpation, such as when uterine rupture immediately occurs after vaginal delivery or when a large tumor mass is present in the vagina.
In most cases, the uterus or tumor can be excised by extrafascial hysterectomy without using this procedure; however, the mastery of total retrograde hysterectomy ${ }^{1-5}$ as an applied procedure is useful.

In principle, when removing a uterus or ovarian tumor with strong adhesions, it is necessary to (1) approach the operation from a site with weak adhesion, (2) avoid approaching only from the same direction, (3) use a procedure with a low complication rate, and (4) pretreat to prevent bleeding before beginning the operation.

The strategy of retrograde hysterectomy is based on the following four principles. (1) The vaginal cavity should be opened at the safest site; (2) A curved Pean forceps should be inserted at the upper end of the vaginal cavity and used as a guide to cut the anterior vaginal wall; (3) Ureteral injury should be avoided by clamping the ligaments in contact with the cervix under direct vision; (4) The posterior vaginal wall should be cut and the uterus removed. By following these principles, the vaginal wall can be safely cut at the upper end of the vagina, and the uterus can be removed without damaging the ureter or rectum.

\section{Surgical Steps}

The surgical procedure involves 10 steps.

1. The round ligament is cut.

$\downarrow$

2. The fallopian tube and ovarian ligament or the infundibulopelvic ligament is cut.
3. The urinary bladder is mobilized.

$\downarrow$

4. The uterine artery and upper part of the cardinal ligament are cut.

$\downarrow$

5. The anterior vaginal wall is incised and the vaginal cavity is opened, and the anterior vaginal wall is then cut at the cervicovaginal junction.

$\downarrow$

6. The bilateral vesicouterine ligaments are cut.

$\downarrow$

7. The bilateral sacrouterine ligaments are cut.

$\downarrow$

8. The posterior vaginal wall is cut and the uterus is removed.

$\downarrow$

9. The vaginal stump is closed.

$\downarrow$

10. Hemostasis is applied.

\section{Selection of Operation Method}

Although the type of operation to be performed can be roughly determined from the preoperative internal examination and imaging diagnosis, the final operation method is decided according to the laparotomy findings as follows:

- No adhesion around the uterus: extrafascial hysterectomy

Copyright $(2019$ by Thieme Medical Publishers, Inc., 333 Seventh Avenue, New York, NY 10001, USA. Tel: +1(212) 584-4662.
License terms

()(1) $\Theta \circledast$ 
- Adhesion around the uterus, but no tumor in the pouch of Douglas: intrafascial hysterectomy (Aldridge method), retrograde hysterectomy

- Tumor in the pouch of Douglas with strong adhesion: retrograde hysterectomy

- Large tumor in the vagina: retrograde hysterectomy

- Cervicovaginal junction is not clear by palpation immediately after vaginal delivery: retrograde hysterectomy

\section{Indications for Retrograde Hysterectomy}

- The adhesion around the uterus is strong and the usual surgical method is impossible

- External endometriosis, chocolate cyst

- Ovarian tumor with strong adhesion in the pouch of Douglas

- History of many surgical operations

- After peritonitis

- The position of the portio vaginalis uteri cannot be determined by palpation during laparotomy

- Patients requiring hysterectomy after vaginal delivery: atonic bleeding, uterine rupture, inversion of uterus, etc.

- Large tumor mass in the vagina: myoma delivery, endometrial stromal sarcoma, tumor of portio vaginalis

\section{Preoperative Preparation}

Internal and external examination, computed tomography, and magnetic resonance imaging should be conducted when adhesions around the uterus are suspected. Intra-venous pyelography should also be performed in such cases to evaluate the presence of adhesions with surrounding organs and assess the position of the ureter.

If uterine rupture results in a retroperitoneal hematoma, a ureteral stent should be inserted. The ureter can be easily found when the stent is inserted, and ureteral damage at the time of pelvic hematoma removal can be avoided.

Additionally, because this surgery is performed in patients with strong adhesions or on an emergency basis after childbirth, autologous blood or preserved blood should be prepared. Sufficient informed consent for the operation method is needed, and preparations should be made for potential complications based on the above findings. In addition to massive bleeding, complications may include damage to the gastrointestinal tract, bladder, and ureter.

\section{Surgical Technique}

The procedure performed to treat an ovarian tumor that has invaded the pouch of Douglas and resulted in a frozen pelvis is described below and illustrated in - Fig. 1 .

\section{Step 1: Cutting of the Round Ligament}

Absorbable suture (No. 1-0) is placed on the round ligament 1.5 to $2.0 \mathrm{~cm}$ away from the uterus, and the ligament is then clamped on its uterus side with a Kocher forceps and cut (-Fig. 2). The incision is slightly expanded upward and downward, and the anterior leaf of the broad ligament is then cut across the vesicouterine peritoneal reflection.

\section{Step 2: Clamping, Cutting, and Ligation of the Fallopian Tube and Ovarian Ligament (or Infundibulopelvic Ligament)}

When preserving the ovary, a Heaney forceps and Kocher forceps are placed on the Fallopian tube and ovarian ligament, and the space between them is cut. Each forceps is then replaced with a ligature. When removing the ovary, the infundibulopelvic ligament is clamped, cut, and ligated. The pelvic side of the ligament is double-ligated, and the ligature is held with a Kocher forceps.

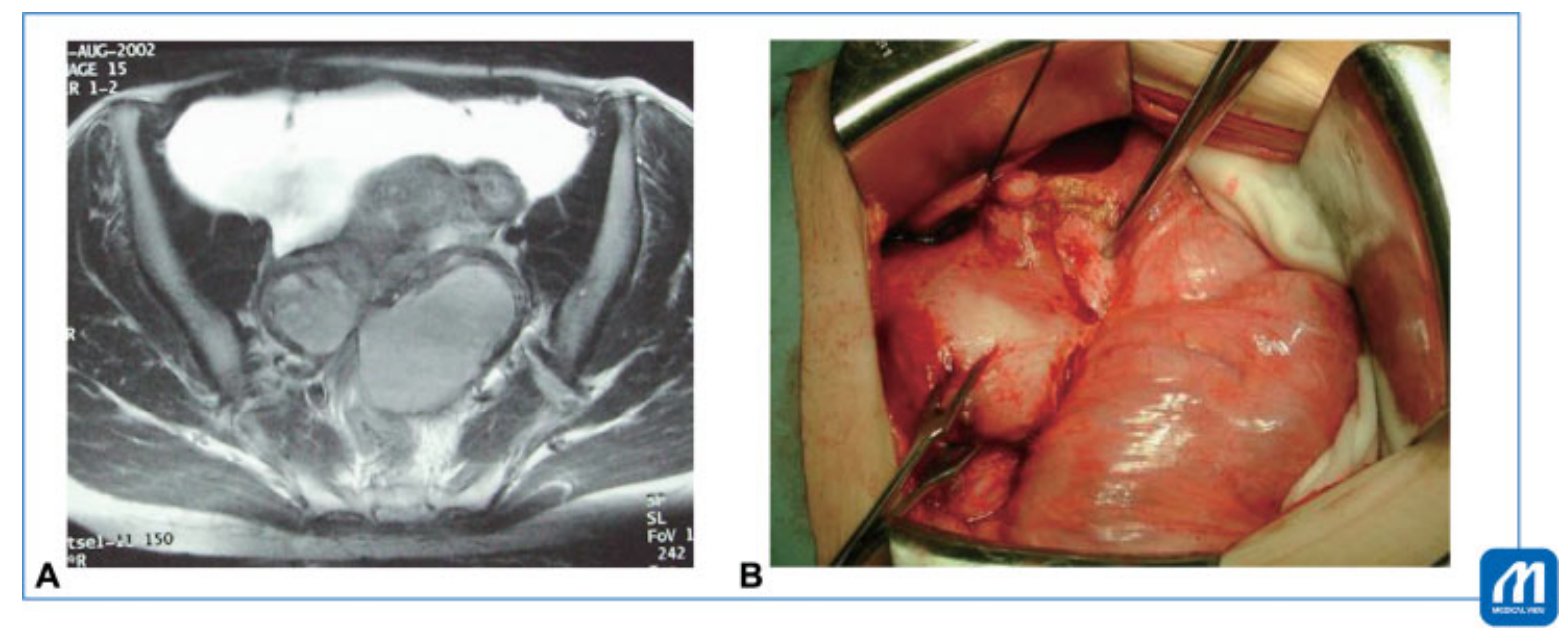

Fig. 1 Magnetic resonance imaging (MRI) and the abdominal findings: MRI revealed bilateral ovarian tumors adhering to the posterior surface of the uterus (A). The abdominal findings showed that the intestinal tract and ovarian tumors adhered to the uterus and became frozen pelvis (B). (Reproduced with permission from 1. Hiramatsu Y. Basic Procedure 1. In: Hiramatsu Y, Konishi I, Sakuragi N, Takeda S, eds. Mastering the Essential Surgical Procedures OGS Now, No. 2 Total Abdominal Hysterectomy (Japanese). Tokyo: Medical View; 2010: 162-169. Copyright $\odot$ Medical View). 
Fig. 2 Cutting of uterine vessels and upper part of cardinal ligament: The uterine vessels and cardinal ligament are clamped at slightly below the internal os of the uterus with a Heaney forceps, and the uterus side is clamped with a Kocher forceps to control back-bleeding from the uterine side. (Reproduced with permission from Hiramatsu. ${ }^{5}$ Y. Basic Procedure 1. In: Hiramatsu Y, Konishi I, Sakuragi N, Takeda S, eds. Mastering the Essential Surgical Procedures OGS Now, No. 2 Total Abdominal Hysterectomy (Japanese). Tokyo: Medical View; 2010: 162-169. Copyright (c) Medical View).

\section{Tips and Warnings}

If the ovarian tumor is adhered to the pelvic floor, the ureter may be damaged during clamping and cutting of the infundibulopelvic ligament. Therefore, the surgeon should confirm that the ureter is not present in the surgical field by direct visualization or palpation.

\section{Step 3: Mobilization of Bladder}

The bladder is mobilized in the same way as during extrafascial abdominal hysterectomy. It is important to start the bladder mobilization at the midline of the cervix to prevent bleeding. Lateral blunt dissection is then performed with Cooper's scissors to expose the vesicouterine ligament.

When the portio vaginalis uteri cannot be felt by palpation, the bladder should be peeled off until reaching the point at 1.5 to $2.0 \mathrm{~cm}$ below the cervicovaginal junction. Even when strong adhesions are present in the pouch of Douglas because of endometriosis or ovarian cancer, adhesion of the vesicouterine pouch is usually not so strong, and mobilization of the bladder is possible.

\section{Step 4: Cutting of Uterine Vessels and Upper Part of Cardinal Ligament}

The uterus is pulled to the upper opposite side, and the posterior lobe of the broad ligament is cut toward the sacrouterine ligament. The loose connective tissue around the cardinal ligament is carefully dissected and removed until the uterine artery meandering above it becomes visible. Next, the uterine vessels and cardinal ligament are clamped at a point slightly below the internal os of the uterus with a Heaney forceps, and the uterus side is clamped with a Kocher forceps to control back-bleeding from the uterine side; these structures are then divided (-Fig. 2). Absorbable suture (No. $1-0$ ) is applied for ligation. The ligature is gripped and pulled with a Kocher forceps. The cut end of the uterine artery must be double-ligated.

Gauze is then placed on this cut end, and the gauze is pinched between the second and third fingers while strongly pulling the uterus upward. The cut end of the uterine vessels and cardinal ligament is then slowly and carefully pushed down to the level of the vesicouterine ligament and sacrouterine ligament.

These procedures (Steps 1-4) are similar to extrafascial hysterectomy (see the section Basic Standard Procedure of Abdominal Hysterectomy: Part 1).

\section{Step 5: Incision of the Anterior Vaginal Wall and Opening of the Vaginal Cavity}

During extrafascial hysterectomy, the sacrouterine ligament and vesicouterine ligament are divided, and the vaginal wall is then cut. However, when retrograde hysterectomy is performed, immediately following Step 4, a longitudinal incision of 3 to $4 \mathrm{~cm}$ is made from the anterior wall of the cervix to the vagina to open the vaginal cavity (-Fig. 3 ). This is done because the position of the portio vaginalis uteri is unknown or because the adhesion of the pouch of Douglas is too strong to mobilize the rectum.

The vaginal cavity can then be found at the lower end of the incision, and its boundary with the portio vaginalis uteri

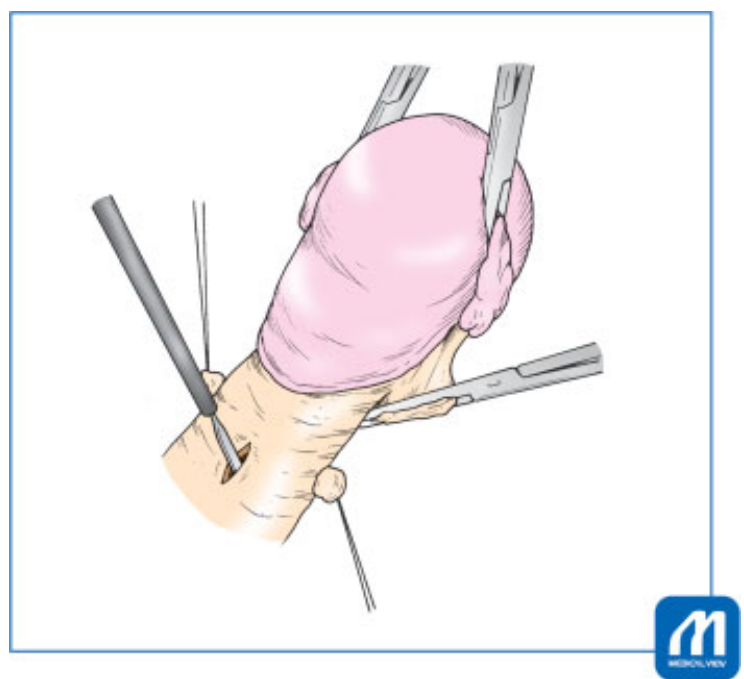

Fig. 3 Incision of the anterior vaginal wall: In case the boundary between the portio vaginalis uteri and the vagina cannot be recognized, a longitudinal incision of 3 to $4 \mathrm{~cm}$ is made from the anterior wall of the uterine cervix to the vagina. (Reproduced with permission from 1. Hiramatsu Y. Basic Procedure 1. In: Hiramatsu Y, Konishi I, Sakuragi N, Takeda S, eds. Mastering the Essential Surgical Procedures OGS Now, No. 2 Total Abdominal Hysterectomy (Japanese). Tokyo: Medical View; 2010: 162-169. Copyright ( Medical View). 
can be recognized ( - Fig. 4). A curved Pean forceps is then inserted along the junction of the portio vaginalis uteri and vaginal wall. This forceps is used as a guide to cut the anterior vaginal wall to the vesicouterine ligament attachment with an electric knife (-Fig. 5). The same incision is performed on the other side, and the inside of the vagina is disinfected.

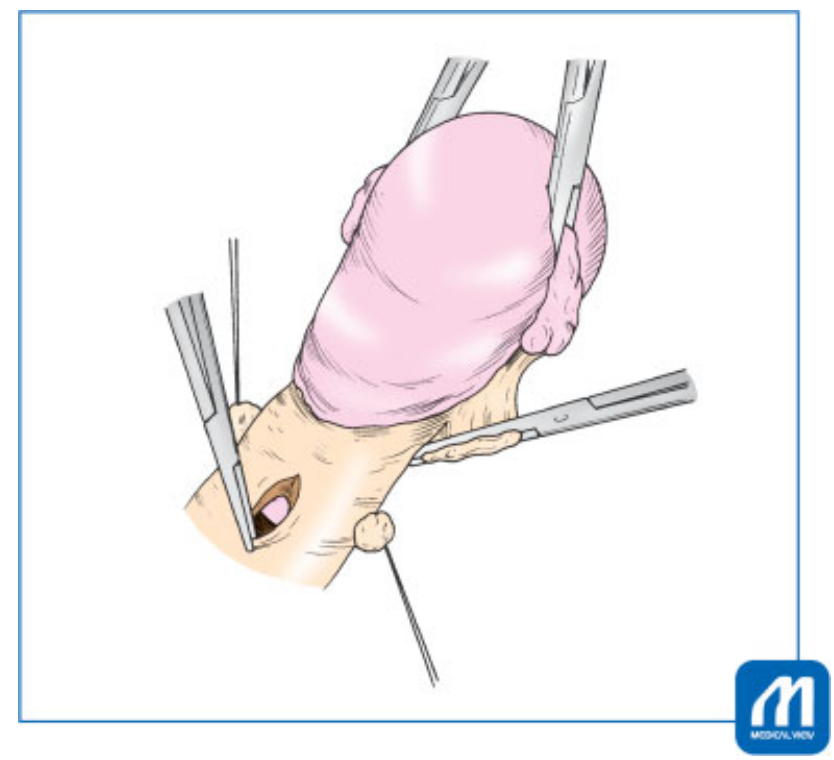

Fig. 4 Opening of the vaginal cavity: The vaginal cavity can be found at the lower end of the incision, and its boundary with the portio vaginalis uteri can be recognized (Reproduced with permission from 1. Hiramatsu Y. Basic Procedure 1. In: Hiramatsu Y, Konishi I, Sakuragi $\mathrm{N}$, Takeda S, eds. Mastering the Essential Surgical Procedures OGS Now, No. 2 Total Abdominal Hysterectomy (Japanese). Tokyo: Medical View; 2010: 162-169. Copyright @ Medical View).

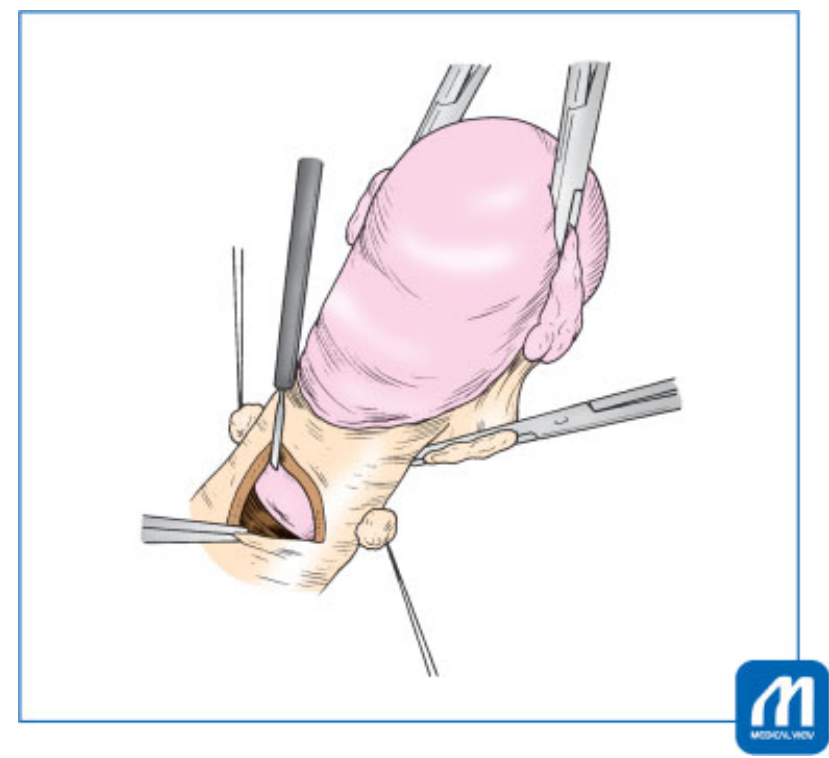

Fig. 5 Cutting the anterior vaginal wall: Cut the anterior vaginal wal at the cervicovaginal junction to the vesicouterine ligament attachment. It is useful to use a curved Pean forceps as a guide to cut the anterior vaginal wall. (Reproduced with permission from 1. Hiramatsu Y. Basic Procedure 1. In: Hiramatsu Y, Konishi I, Sakuragi N, Takeda S, eds. Mastering the Essential Surgical Procedures OGS Now, No. 2 Total Abdominal Hysterectomy (Japanese). Tokyo: Medical View; 2010: 162-169. Copyright (c) Medical View).

\section{Tips and Warnings}

The above-described technique is performed when the boundary between the portio vaginalis uteri and vagina cannot be determined by palpation. If this boundary can be felt by palpation, a transverse incision at the border of the portio vaginalis uteri rather than a longitudinal incision in the center of the cervix makes subsequent procedures easier.

\section{Step 6: Clamping, Cutting, and Ligation of the Bilateral Vesicouterine Ligaments}

Next, as shown in - Fig. 6, a single leaf of the Heaney forceps is inserted into the vagina, and the vesicouterine ligament and the anterior half of the cardinal ligament are then clamped in contact with the portio vaginalis uteri and divided. The forceps is then replaced with 1-0 absorbable suture.

It is important to use the Heaney forceps in such a way that the convex surface of the forceps points diagonally forward of the uterus; that is, in the direction in which the vesicouterine ligament is attached.

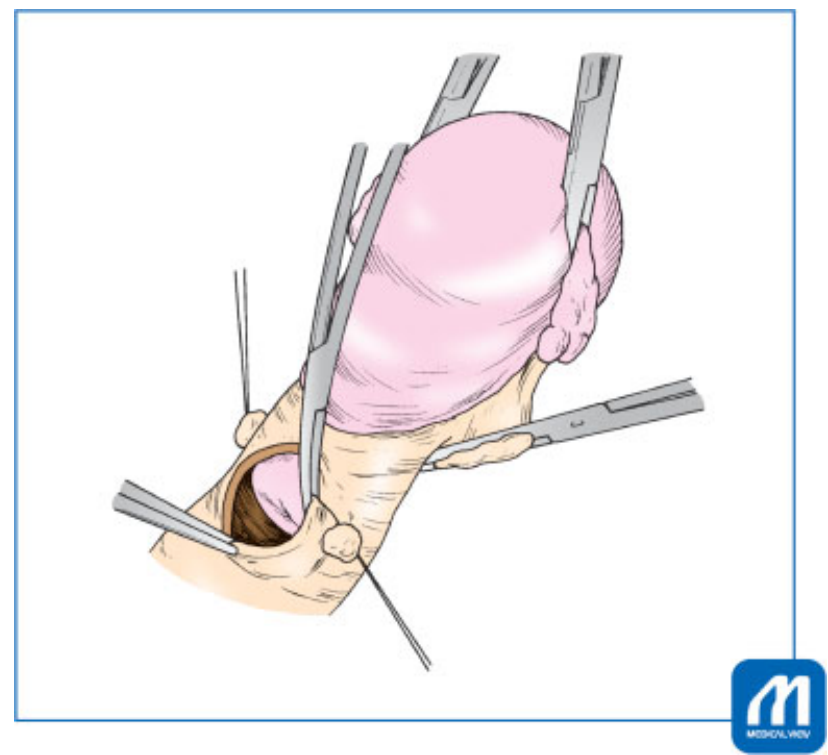

Fig. 6 Cutting the bilateral vesicouterine ligaments: A single leaf of the Heaney forceps is inserted into the vagina, then the vesicouterine ligament and the anterior half of the cardinal ligament are clamped in contact with the portio vaginalis uteri and divided. (Reproduced with permission from 1. Hiramatsu Y. Basic Procedure 1. In: Hiramatsu Y, Konishi I, Sakuragi N, Takeda S, eds. Mastering the Essential Surgical Procedures OGS Now, No. 2 Total Abdominal Hysterectomy (Japanese). Tokyo: Medical View; 2010: 162-169. Copyright @ Medical View). 


\section{Tips and Warnings}

This operation method is performed without directly visualizing the ureter, so appropriate use of the Heaney forceps is very important. The following two precautions are necessary to avoid ureteral damage and ensure that the uterine ligaments are cut along the round aspect of the uterine cervix. First, a single leaf of the Heaney forceps is inserted into the vagina in close contact with the boundary between the portio vaginalis uteri and vaginal wall. Second, the convex surface of the Heaney forceps is directed obliquely forward of the uterus, and clamping is performed. Importantly, the forceps must be oriented in this direction and be placed in contact with the portio vaginalis uteri.

\section{Step 7: Clamping, Cutting, and Ligation of the Bilateral Sacrouterine Ligaments}

A single leaf of the Heaney forceps leaf is inserted into vagina so that it is placed in contact with the portio vaginalis uteri, and the posterior parts of the cardinal ligament and sacrouterine ligament are clamped. At this time, when cutting the left side, the Heaney forceps should face diagonally left and backward of the uterus ( - Fig. 7); the ligaments can thus be clamped in the correct direction, and ureteral damage can be avoided. The ligaments are cut and ligated with $1-0$ absorbable suture.

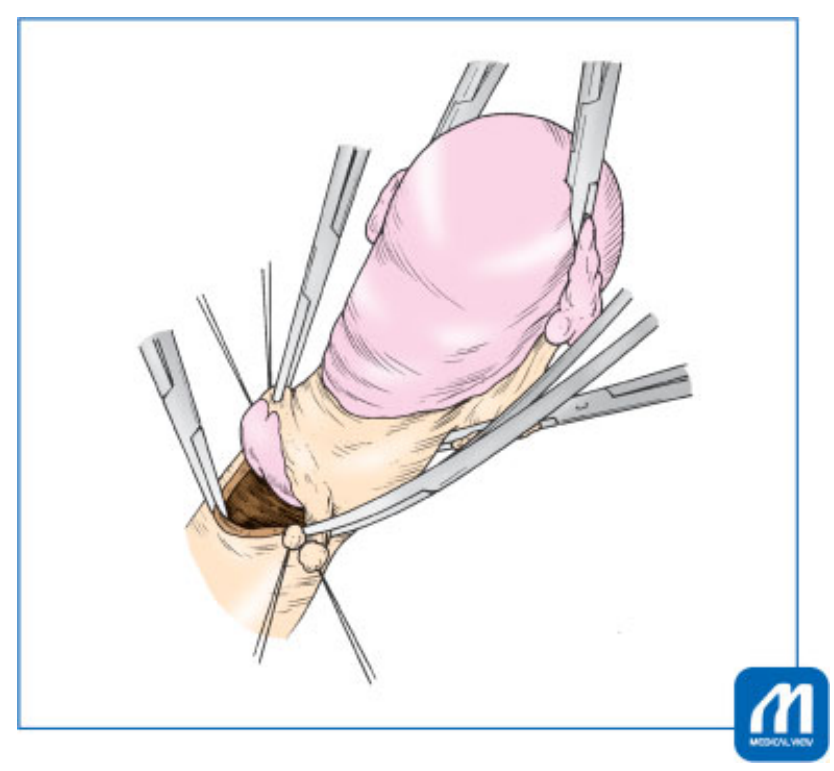

Fig. 7 Cutting the bilateral sacrouterine ligaments: A single leaf of the Heaney forceps leaf is inserted into vagina so that it is placed in contact with the portio vaginalis uteri, and the posterior part of the cardinal ligament and sacrouterine ligament are clamped. (Reproduced with permission from 1. Hiramatsu Y. Basic Procedure 1. In: Hiramatsu Y, Konishi I, Sakuragi N, Takeda S, eds. Mastering the Essential Surgical Procedures OGS Now, No. 2 Total Abdominal Hysterectomy (Japanese). Tokyo: Medical View; 2010: 162-169. Copyright $\odot$ Medical View).

\section{Step 8: Incision of the Posterior Vaginal Wall}

If the above operation is also performed on the contralateral side, the uterus is connected only by the posterior vaginal wall. Even when strong adhesions are present in the pouch of Douglas, it is easy to cut the posterior vaginal wall and avoid rectal injury

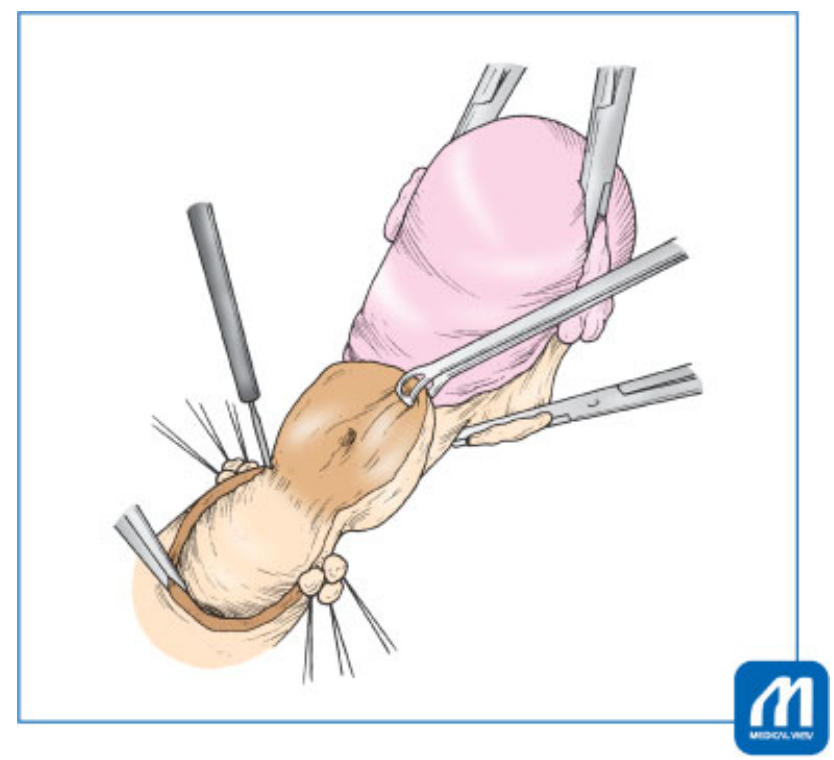

Fig. 8 Incision of the posterior vaginal wall: The uterus is connected only by the posterior vaginal wall. Cut the posterior vaginal wall carefully. (Reproduced with permission from 1. Hiramatsu Y. Basic Procedure 1. In: Hiramatsu Y, Konishi I, Sakuragi N, Takeda S, eds. Mastering the Essential Surgical Procedures OGS Now, No. 2 Total Abdominal Hysterectomy (Japanese). Tokyo: Medical View; 2010: 162-169. Copyright @ Medical View).

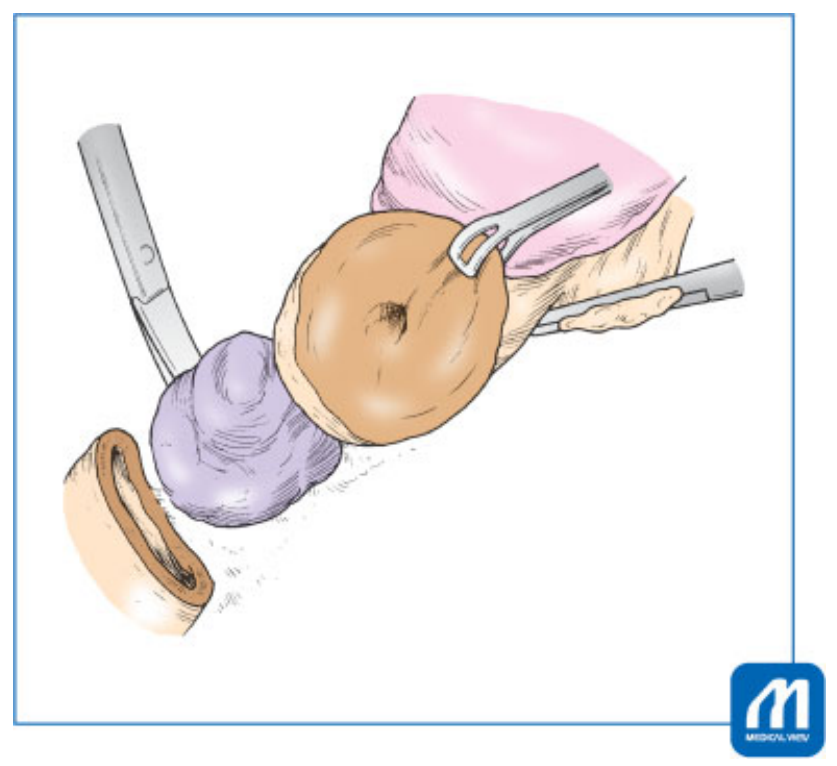

Fig. 9 In case of ovarian tumor of Douglas pouch with severe adhesion: When the posterior vaginal wall is cut and the uterine cervix is liberated, it is possible to peel the tumor of Douglas pouch from the lower side and even remove a tumor with severe adhesions. The uterus and the ovarian tumor can be removed as one mass without peeling the adhesion between the posterior uterine wall and the tumor. (Reproduced with permission from 1 . Hiramatsu Y. Basic Procedure 1. In: Hiramatsu Y, Konishi I, Sakuragi N, Takeda S, eds. Mastering the Essential Surgical Procedures OGS Now, No. 2 Total Abdominal Hysterectomy (Japanese). Tokyo: Medical View; 2010: 162-169. Copyright $\odot$ Medical View). 


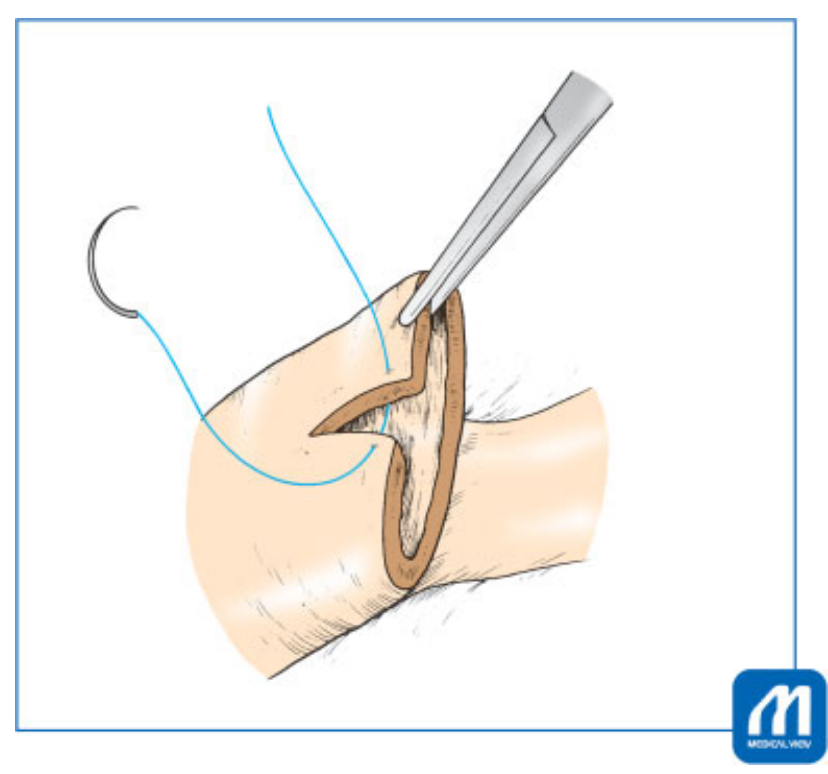

Fig. 10 Closing of the vaginal vault: After the uterus has been removed, the inside of the vagina is disinfected and the vaginal canal is closed. (Reproduced with permission from 1. Hiramatsu Y. Basic Procedure 1. In: Hiramatsu Y, Konishi I, Sakuragi N, Takeda S, eds. Mastering the Essential Surgical Procedures OGS Now, No. 2 Total Abdominal Hysterectomy (Japanese). Tokyo: Medical View; 2010 : 162-169. Copyright (c) Medical View).

because the position of the uterine cervix is clear at this point (-Fig. 8).

If an ovarian tumor is present in the pouch of Douglas, the posterior vaginal wall should be carefully incised to avoid rupturing the tumor. When the posterior vaginal wall is cut and the uterine cervix is liberated, it is possible to peel the tumor from the lower side and even remove a tumor with severe adhesions (-Fig.9). The uterus and the ovarian tumor can be removed as one mass without peeling the adhesion between the posterior uterine wall and the tumor.

\section{Step 9: Closing of the Vaginal Vault}

After the uterus has been removed, the inside of the vagina is disinfected and the vaginal canal is closed. First, sutures are knotted on the bilateral ends of the vagina, and the longitudinal incision of the anterior vaginal wall is repaired. Continuous

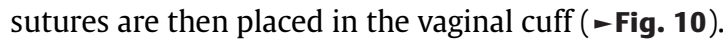

\section{Step 10: Hemostasis and Closure of the Abdominal Wall}

If a wide adhesion release surface is present and the surgeon is concerned about postoperative bleeding, an information drain should be inserted. A gauze count is performed and the abdominal wall is closed.

Conflict of Interest

None declared.

\section{Acknowledgment}

We thank Angela Morben, DVM, ELS, from Edanz Group (www.edanzediting.com/ac), for editing a draft of this manuscript.

\section{References}

1 Sekiba K, Hiramatsu Y. Retrograde abdominal hysterectomy. Therapy of Obstetrics and Gynecology(Japanese) 1991;62:267-273

2 Hiramatsu Y, Sekiba K. Retrograde abdominal hysterectomy. In Practical Methods for Hysterectomy. Tokyo: Nankodo; 1997:61-77

3 Hiramatsu Y, Kudo T. Application of retrograde abdominal hysterectomy and ligation of internal iliac artery in advanced uterine tumor surgery. Obstet Gynecol Surgery(Japanese) 2003;14:145-151

4 Hiramatsu Y. Application of retrograde abdominal hysterectomy in debulking surgery of epithelial ovarian cancer. Obstet Gynecol (Japanese) 2009;76:409-415

5 Hiramatsu Y. Basic procedure 1. In Hiramatsu Y, Konishi I, Sakuragi N, Takeda S, eds. Mastering the Essential Surgical Procedures OGS Now, No.2 Application of Retrograde Abdominal Hysterectomy. Tokyo: Medical View; 2010:162-169 